\title{
Indumento y tricomas en la caracterización de un grupo de nueve especies del género Mortoniodendron (Tiliaceae)
}

\author{
Indument and trichomes in the characterization of a group of nine species of Mortoniodendron \\ (Tiliaceae)
}

\author{
Nelson Rendón-Carmona ${ }^{1}$, Mario Ishiki-Ishihara ${ }^{1}$, Teresa Terrazas ${ }^{2 *}$ y María Guadalupe Nieto-López ${ }^{3}$ \\ ${ }^{1}$ El Colegio de la Frontera Sur, Unidad San Cristóbal, Carretera Panamericana y Periférico Sur S/N C.P. 29290, San Cristóbal de Las Casas, \\ Chiapas. \\ ${ }^{2}$ Programa de Botánica, Colegio de Postgraduados, Carretera México-Texcoco, km 36.5, Montecillo, Estado de México, C. P. 56230. Dirección actual: \\ Departamento de Botánica, Instituto de Biología, Universidad Nacional Autónoma de México. Apartado postal 70-233, 04510 México D.F. \\ ${ }^{3}$ Laboratorio MEB. El Colegio de la Frontera Sur, Unidad Tapachula, Carretera Antiguo Aeropuerto, Km. 2.5, C.P. 30700, Tapachula, Chiapas. \\ *Correspondencia: tterrazas@ibiologia.unam.mx
}

Resumen. Se estudió el indumento y la morfología de los tricomas del envés de las hojas y del endocarpo de 9 especies del género Mortoniodendron (Tiliaceae). Se establece el tipo de indumento y de tricomas según la forma, simetría, tamaño y densidad, mediante microscopía electrónica de barrido y óptica. En las hojas y endocarpo se definen 2 categorías para el tipo de indumento. Para las hojas, el axilar se presenta en 7 especies y glabrescente en 9 y para el endocarpo glabrescente en 5 especies y el resto glabras. Con relación a la forma, simetría y tamaño se reconocen 4 tipos de tricomas: simples de un brazo, fasciculados de 2 a 5 brazos, estrellados de 3 a 18 brazos y capitados uni y multicelulares. Los tricomas simples y fasciculados predominan en $M$. costaricense, $M$. hirsutum y $M$. vestitum. Los estrellados están presentes en todas las especies y se distinguen 4 categorías según el tamaño y número de brazos. Sólo en M. palaciosii y M. ruizii se encuentran tricomas capitados. En el endocarpo predominan los tricomas estrellados, excepto en $M$. vestitum con tricomas fasciculados largos. Se discute la importancia taxonómica de los resultados y se elabora una clave para identificar las especies.

Palabras clave: endocarpo, hojas, Mortoniodendron, Tiliaceae, tricomas.

\begin{abstract}
The indument and trichome morphology in leaves and endocarp for 9 species of Mortoniodendron (Tiliaceae) were studied. The distribution and the type of trichomes were established according to their form, symmetry, size, and density using scanning electron and optical microscopy. Two categories for the distribution of trichomes were defined in the leaves and the endocarp. In the case of the leaves, 7 species have axillar indument and it is glabrescent in 9, while in the endocarp 5 species show glabrescent and others glabrous. Four types of trichomes were recognized: acicular with a single arm, fasciculate with 2-5 arms, stellate with 3-18 arms, and uni or multicellular capitate. Acicular and fasciculate trichomes prevail in $M$. costaricense, $M$. hirsutum and $M$. vestitum. Stellate trichomes are present in all the species, but 4 categories are distinguished according to size and number of arms. Only M. palaciosii and M. ruizii possess capitate trichomes. In the endocarp stellate trichomes prevail, except for $M$. vestitum with long fasciculate trichomes. The taxonomic usefulness of the results is discussed and a key to identify the species is provided.
\end{abstract}

Key words: endocarp, leaves, Mortoniodendron, Tiliaceae, trichomes.

\section{Introducción}

El indumento es un atributo de la mayoría de las angiospermas; sin embargo, puede o no estar presente en todos los órganos de un individuo. El estudio de la

Recibido: 22 agosto 2005; aceptado: 03 mayo 2006 morfología y distribución de los tricomas genera evidencias importantes sobre las relaciones a diferentes niveles de la clasificación de angiospermas (Dickison, 2000). Además, se considera de valor taxonómico el reconocimiento de los diferentes tipos de tricomas, más que su sola presencia o ausencia (Theobald et al., 1988).

Los tricomas, como unidad elemental del indumento, son importantes en estudios sistemáticos, fisiológicos 
y ecológicos. Según su función, se han reconocido tres grupos: como adaptaciones al medio físico, como mecanismo de defensa físico-químico contra patógenos o depredadores y como parte del control fisiológico en el intercambio de productos entre la planta y el ambiente (Gual, 1998).

Para evaluar la importancia del indumento y tricomas como herramienta taxonómica es necesario reconocer las variaciones estocásticas espaciales o temporales, de naturaleza geográfica, ecológica o estacional. La densidad debe considerar posibles variaciones relacionadas con la posición relativa sobre la hoja, dado que en determinadas zonas, pecíolo, base, ápice, venas, axilas o margen, el indumento puede presentar un comportamiento diferente.

La forma, densidad y coloración del indumento se han utilizado como caracteres taxonómicos para la separación de especies en los géneros Luehea, Tilia, Trichospermum (Gual, 1998). En especies del género Mortoniodendron, diversos autores mencionan la presencia de tricomas estrellados en la epidermis abaxial de las hojas, sin especificar el número de brazos, el tamaño o la simetría de los mismos (Standley y Steyermark, 1940; Lundell, 1965; Dorr y Wendt, 2004; Rodríguez, 2004). Asimismo, Standley (1942) resalta la presencia de abundantes tricomas simples, largos y rectos en $M$. hirsutum. Por otra parte, Bamps y Robyns (1977) citan como glabra en las hojas y en el endocarpo a Westphalina macrocarpa A. Robyns et Bamps [= Mortoniodendron pentagonum (Donn. Sm.) Miranda]. Miranda (1956) observa tricomas estrellados en el interior del ovario de M. guatemalense, pero ausentes en M. palaciosii Miranda. Recientemente, Dorr y Wendt (2004) también reconocen el interior del fruto de $M$. uxpanapense Dorr et T. Wendt como glabro.

El propósito del presente estudio es describir el tipo de indumento y tricomas presentes en las hojas y el endocarpo de 9 especies del género Mortoniodendron mediante el análisis de imágenes generadas por el microscopio electrónico de barrido, así como construir una clave que ayude a la identificación de las especies.

\section{Material y método}

Se revisaron 200 ejemplares correspondientes a 9 especies del género Mortoniodendron, provenientes de los herbarios MEXU, ENCB, XAL, CHAPA y ECOSUR. La lista completa de ejemplares revisados se puede solicitar al primer autor. En el apéndice se listan únicamente los materiales examinados en el microscopio electrónico de barrido (MEB).

Para evaluar el tipo de indumento o tricomas, en cada ejemplar se observaron de 3 a 5 láminas por el envés y en casi todos los casos, las observaciones del pecíolo corresponden a la región basal de la lámina. El tipo y tamaño de los tricomas se determinó con base en el análisis de imágenes obtenidas en el MEB (Topcon SM510). Para su observación, las muestras se recortaron en cuadros de $3.0 \mathrm{~mm}$ de lado de la epidermis abaxial de la hoja (base, media y ápice) y endocarpo; se fijaron mediante un pegamento a base de carbono y se metalizaron con oropaladio. Además, automáticamente se produjeron fotos de todas las especies en formato digital con el MEB.

Para designar el tipo de indumento y tricomas de cada especie se utilizaron 8 variables; 5 cualitativas: forma, distribución, predominancia, simetría y tipo de crecimiento de tricomas, y 3 cuantitativas: densidad promedio, tamaño y número de brazos. Se consideraron predominantes aquellos tricomas con mayor presencia $(\geq 70 \%$ ) que el resto, y escasos los presentes en menor proporción $(<30 \%)$. La simetría se definió en 2 grupos: axial, por reflexión en un plano, el cual divide longitudinalmente al tricoma unicelular y radial en tricomas multicelulares, donde el largo de los brazos y la separación angular entre éstos es similar; es decir, con simetría de rotación dextrógira respecto a un eje propio. El tipo de crecimiento se definió respecto al plano en que se encuentran insertos. El orden de la nervación se define según el criterio de la ramificación y grosor; por ejemplo, a partir de la nervadura central y laterales de primer orden, se establecen los órdenes subsecuentes (Hickey, 1988). La densidad promedio se estimó sin distinción del tipo de tricoma, se efectuó un conteo de los mismos en 3 zonas abaxiales de la hoja: base, parte media y ápice, en un cuadro de $4 \mathrm{~mm}^{2}$.

La distribución de los tricomas se estableció en 4 grupos, considerando las definiciones de Valencia y Delgado (2003), 2 de ellos modificados para expresar la diversidad encontrada en Mortoniodendron: axilar, los tricomas definen un perímetro en las convergencias de los nervios primarios y secundarios o de orden superior, alrededor de los domacios; glabra, no presenta tricomas; glabrescente, los tricomas se encuentran dispersos regularmente en todo el envés o en zonas como la base, los nervios y el margen de la hoja, así como en la superficie del endocarpo, de tal manera que es posible apreciar la epidermis; pelosa, los tricomas cubren totalmente la superficie del envés de la hoja o la superficie del endocarpo, lo cual impide apreciar la epidermis.

La forma de los tricomas se designó, según la clasificación de Hardin (1990) y Moreno (1984), en 4 tipos: simples, estrellados, fasciculados y capitados. A excepción de los tricomas capitados, los restantes se dividieron en 2 ó 3 categorías, según el tipo de tricoma y la longitud de sus brazos (cortos, medianos y largos). 


\section{Resultados}

Se reconocieron 2 grupos de especies de acuerdo con el tipo de indumento en la hoja. El grupo glabrescente (9 especies) y el axilar (7 especies). El indumento axilar se localizó en distintos órdenes de la nervadura, según la especie, y el indumento glabrescente se presentó en distintas zonas de la lámina (Cuadro 1).

Se encontraron 4 tipos de tricomas. Los simples unicelulares con un brazo recto terminado en punta y espesor poco variable, excepto en el ápice, presentan crecimiento más o menos vertical, simetría axial y 3 categorías correspondientes con el largo del brazo: cortos, medianos y largos (Figs. 1-3). Los fasciculados presentan crecimiento oblicuo con una inclinación de 40 a $70^{\circ}$, simetría radial, compuestos de 2 a 5 brazos; se distinguen 2 categorías: cortos (Fig. 4) y largos, en los cuales ocasionalmente se observa la presencia de estípite (Fig. 5). Los estrellados tienen crecimiento horizontal, simetría radial y el número de brazos varía; de 8 a 14 resultaron los más frecuentes y los de 4 a 7 (Fig. 6) o de 15 a 18 brazos son poco frecuentes, el umbo es distintivo y su superficie aumenta con el número de brazos; se reconocieron 2 categorías: cortos y largos. Los capitados con estípite y ápice oblongo presentan crecimiento vertical u oblicuo, simetría axial, el número de células en el ápice varía de 1 a 15 (Fig. 7).

La predominancia de algunos tipos de tricomas de la lámina distingue a cada especie o grupos de éstas (Cuadro 2). Los tricomas simples cortos se encontraron con abundancia sobre las nervaduras de $M$. ruizii y, en menor proporción, en el pecíolo de $M$. pentagonum; los medianos, en toda la superficie abaxial de $M$. vestitum y en 8 de las especies alrededor de los domacios; resultaron escasos en $M$. anisophyllum y $M$. guatemalense. Los simples largos se presentaron exclusivamente en las nervaduras de $M$. hirsutum. Los tricomas fasciculados se observaron predominantes en $M$. costaricense Standl. et L. O. Williams y $M$. vestitum, en esta última resultaron más largos; ocasionalmente se encontraron en $M$. anisophyllum y M. guatemalense. Los tricomas estrellados se observaron en todas las especies; con mayor presencia en $M$. anisophyllum, M. guatemalense, M. palaciosii, $M$. pentagonum, $M$. ruizii y $M$. uxpanapense, mientras que en $M$. costaricense, $M$. hirsutum y $M$. vestitum fueron escasos. Lo tricomas capitados fueron exclusivos de $M$. palaciosii y $M$. ruizii, pero son raros. Algunas especies tuvieron una mayor variedad de tricomas, encontrándose tricomas de 3 tipos en 6 especies y de 1 a 2 tipos en 3 especies (Cuadro 2).

De acuerdo con la densidad es posible establecer 3 grupos: el más común tiene una variación abruptamente decreciente de la base al ápice; en este grupo se encuentran M. anisophyllum, M. costaricense, $M$. guatemalense y $M$. hirsutum. Sólo $M$. vestitum presentó una densidad uniforme de la base al ápice. En el grupo con densidad baja se ubicó a $M$. palaciosii, $M$. pentagonum y $M$. uxpanapense. En $M$. ruizii no se estimó la densidad (Cuadro 2).

El indumento en el endocarpo se observó glabrescente a axilar en 5 especies $M$. anisophyllum, M. costaricense, M. guatemalense, M. palaciosii y M. vestitum (Figs. 8-10), mientras que las especies $M$. hirsutum, $M$. pentagonum y $M$. ruizii resultaron glabras ( $M$. uxpanapense se observó como glabro por Dorr y Wendt, 2004). Los tricomas estrellados estuvieron presentes con mayor frecuencia (Cuadro 3); sólo en $M$. vestitum se encontraron tricomas simples y fasciculados en el endocarpo (Fig. 9).

Cuadro 1. Indumento presente en la epidermis abaxial de la hoja en 9 especies de Mortoniodendron. base (b), lámina (1), margen $(\mathrm{m})$, nervadura $(\mathrm{n})$.

\begin{tabular}{|c|c|}
\hline $\begin{array}{c}\text { Axilar } \\
\text { (orden de nervación) }\end{array}$ & $\begin{array}{l}\text { Glabrescente } \\
\text { (región de la lámina) }\end{array}$ \\
\hline $\begin{array}{l}\text { M. anisophyllum (1-5) } \\
\text { M. costaricense }(1-4) \\
\text { M. guatemalense }(1-4) \\
\text { M. hirsutum }(1-3) \\
\text { M. palaciosii }(1-3) \\
\text { M. ruizii }(1-3) \\
\text { M. vestitum }(1-3)\end{array}$ & $\begin{array}{l}\text { M. anisophyllum }(1, \mathrm{~b}, \mathrm{n}) \\
\text { M. costaricense }(\mathrm{b}-\mathrm{n})^{* *} \\
\text { M. guatemalense }(\mathrm{b}, \mathrm{b}-\mathrm{n})^{* *} \\
\text { M. hirsutum }(\mathrm{n}, \mathrm{m}) \\
\text { M. palaciosii }(\mathrm{l})^{*} \\
\text { M. pentagonum }(\mathrm{b})^{*} \\
\text { M. ruizii }(\mathrm{n}) \\
\text { M. uxpanapense }(\mathrm{b})^{*} \\
\text { M. vestitum }(\mathrm{l}, \mathrm{m})\end{array}$ \\
\hline
\end{tabular}

*Cuando presentes, muy raros.

**La zona con indumento varía dependiendo del ejemplar examinado. 


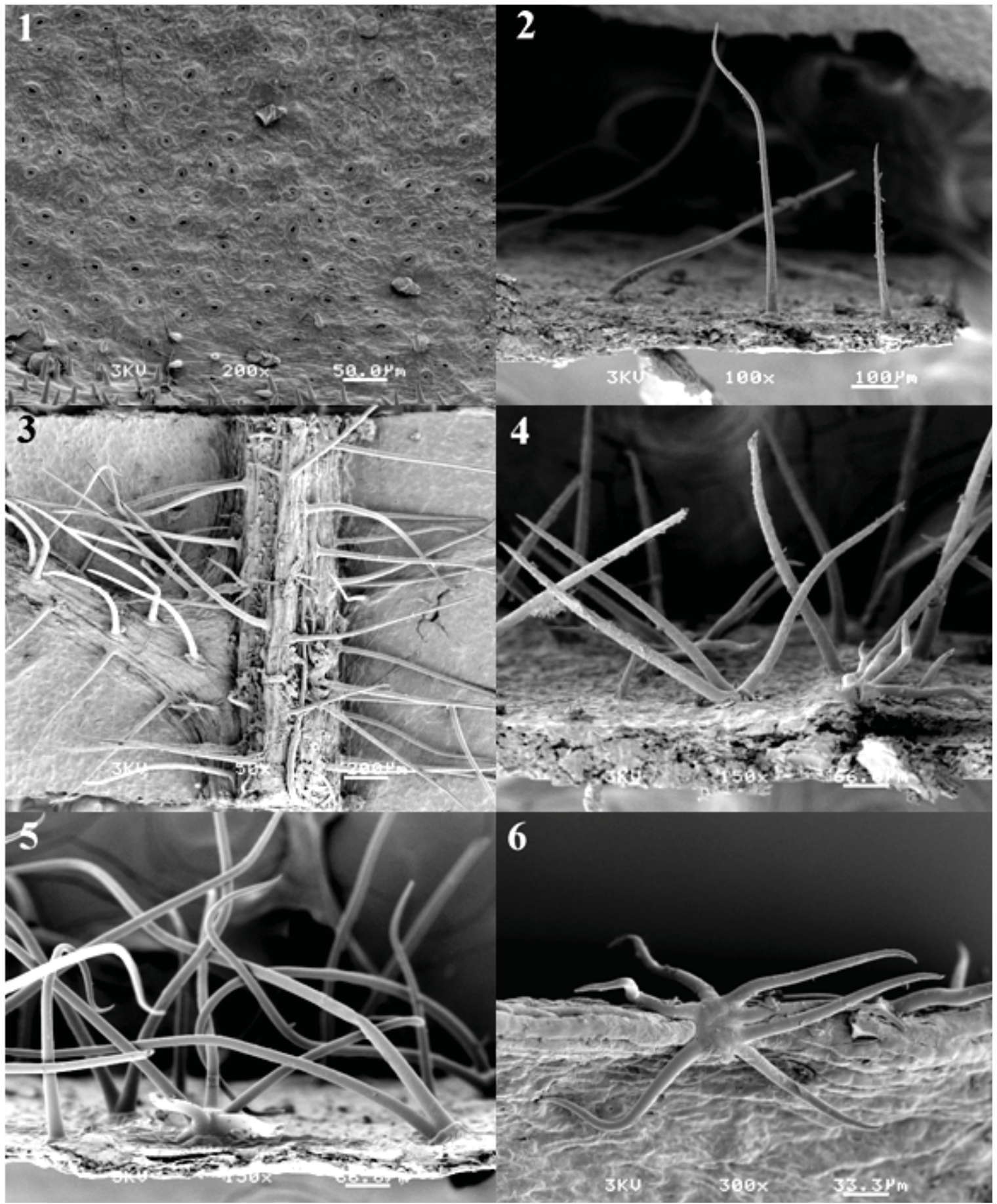

Figuras 1-6. Tipos de tricomas en la epidermis abaxial de las hojas utilizando MEB. 1. Simples cortos en M. ruizii, J. Chavelas 1462 (MEXU). 2. Simples medianos en M. vestitum, E. Martínez 10949 (MEXU). 3. Simples largos en M. hirsutum, J. Meave 1459 (MEXU). 4. Fasciculados cortos en M. costaricense, II Inbio 109 (MEXU). 5. Fasciculados largos en M. vestitum, E. Martínez 8506 (MEXU). 6. Estrellado en M. guatemalense, A. Méndez Ton 6612 (MEXU).

\section{Discusión}

Los resultados permitieron reconocer patrones de importancia taxonómica relacionados con el indumento, densidad, tipo, predominancia, tamaño y número de brazos en los tricomas asociados con especies del género 

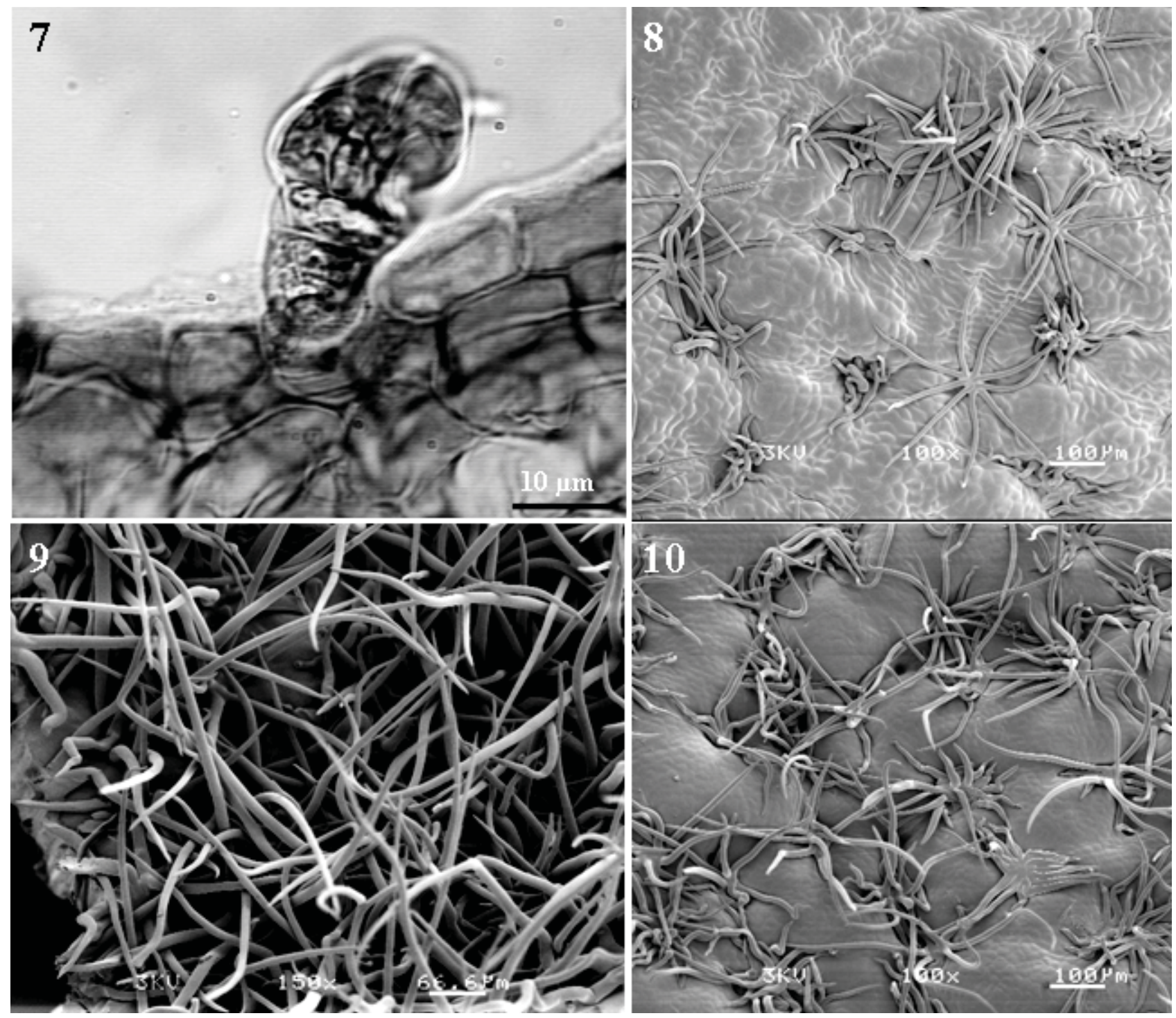

Figuras 7-10. Tipos de tricomas en la epidermis abaxial de las hojas y el endocarpo utilizando MEB. 7. Capitado en M. palaciosii, T. Wendt 6016 (TEX). 8-10. Tipos de tricomas en el endocarpo. 8. Estrellado en M. anisophyllum, J. Solomón 19265 (MEXU). 9. Simples y fasciculados en M. vestitum, J. López-García 92 (ECOSUR). 10. Estrellado en M. guatemalense, C. Gutiérrez 3769 (MEXU).

Mortoniodendron. La simetría axial o radial y el tipo de crecimiento de los tricomas permiten una descripción objetiva y facilitan la identificación de las especies.

La observación cualitativa de Standley y Steyermark (1949) con relación al indumento, definido como casi glabro o basal pubescente, se confirmó en la mayoría de las especies. No se encontraron especies glabras; las especies con menos indumento son M. palaciosii, M. pentagonum у M. uxpanapense. Mortoniodendron uxpanapense se distinguió por el pecíolo peloso y por el indumento que disminuye más rápidamente sobre la base de la vena media, casi glabra fuera de ésta. En el resto de las especies el indumento y los tricomas presentes en el pecíolo resultaron similares a los observados en la parte basal de la nervadura central.

Los 4 tipos de tricomas presentes en Mortoniodendron corresponden con los registrados por Hardin (1990), para las 4 variedades de Tilia americana s. 1., simples, fasciculados, estrellados y capitados. La semejanza con Tilia se hace patente en otras características morfológicas, como la presencia de abundante mucílago (Miranda, 1956), estambres basifijos, sépalos libres, gineceo plurilocular, ovario sincárpico, fruto indehiscente con o sin costillas marcadas, polen oblato, elipsoidal en vista 
Cuadro 2. Tricomas en la epidermis abaxial de la hoja en nueve especies de Mortoniodendron.

\begin{tabular}{|c|c|c|c|c|c|}
\hline \multirow{2}{*}{ Especie } & \multicolumn{3}{|c|}{ Predominantes } & \multicolumn{2}{|c|}{ Escasos } \\
\hline & Tricomas & $\begin{array}{l}\text { Número de } \\
\text { brazos }\end{array}$ & $\begin{array}{l}\text { Longitud } \\
\qquad(\mu \mathrm{m})\end{array}$ & Tricomas & $\begin{array}{l}\text { Número } \\
\text { de brazos }\end{array}$ \\
\hline M. anisophyllum & Estrellados largos & $5-11$ & $100-200(233)$ & $\begin{array}{l}\text { Simples medianos, } \\
\text { fasciculados }\end{array}$ & $\begin{array}{c}1 \\
--\end{array}$ \\
\hline M. costaricense & $\begin{array}{l}\text { Simples medianos, } \\
\text { fasciculados cortos }\end{array}$ & $\begin{array}{c}1 \\
2-4\end{array}$ & $\begin{array}{l}200-400 \\
200-400\end{array}$ & Estrellados cortos & $5-8$ \\
\hline M. guatemalense & Estrellados cortos & (4) $8-14(17)$ & (50) 100-150 & $\begin{array}{l}\text { Simples medianos, } \\
\text { fasciculados }\end{array}$ & $\begin{array}{l}1 \\
2-4\end{array}$ \\
\hline M. hirsutum & Simples largos & 1 & $500-1200$ & Estrellados largos & $4-9$ \\
\hline M. palaciosii & Estrellados cortos & 8 & $50-100$ & $\begin{array}{l}\text { Simples medianos, } \\
\text { capitados }\end{array}$ & $\begin{array}{c}1 \\
6-15\end{array}$ \\
\hline M. pentagonum & $\begin{array}{l}\text { Estrellados cortos, } \\
\text { simples cortos }\end{array}$ & $\begin{array}{c}3-7(10) \\
1\end{array}$ & $\begin{array}{c}60-100 \\
30-50\end{array}$ & -- & -- \\
\hline M. ruizii & $\begin{array}{l}\text { Simples cortos*, } \\
\text { estrellados cortos }\end{array}$ & $\begin{array}{c}1 \\
5-10\end{array}$ & $\begin{array}{c}25-75 \\
100-150\end{array}$ & $\begin{array}{l}\text { Simples medianos, } \\
\text { capitados }\end{array}$ & $\begin{array}{l}1 \\
1\end{array}$ \\
\hline M. uxpanapense & Estrellados cortos & 8,10 & $50-75$ & -- & -- \\
\hline M. vestitum & $\begin{array}{l}\text { Simples medianos, } \\
\text { fasciculados largos }\end{array}$ & $\begin{array}{c}1 \\
2-5\end{array}$ & $\begin{array}{c}200-400 \\
(300) 400-900\end{array}$ & Estrellados cortos & $4-10$ \\
\hline
\end{tabular}

*Estuvieron presentes dependiendo del ejemplar examinado.

** La densidad se refiere sólo a las nervaduras; el resto del envés resultó casi glabro

Cuadro 3. Tipo de tricomas presentes en el endocarpo en 8 especies de Mortoniodendron.

\begin{tabular}{lccc}
\hline Especie & Tricomas & Número de rayos & Longitud $(\mu \mathrm{m})$ \\
\hline M. anisophyllum & Estrellados & $6-10$ & $100-200$ \\
M. costaricense & Estrellados & $6-8$ & $200-400$ \\
M. guatemalense & Estrellados & $6-12$ & $200-400(500)$ \\
$M$. hirsutum & Glabro & -- & -- \\
M. palaciosii* & Estrellados & No determinado & No determinado \\
M. pentagonum & Glabro** & -- & -- \\
M. ruizii & Glabro & -- & -- \\
$M$. uxpanapense & Glabro** & -- & -- \\
$M$. vestitum & Fasciculados largos & $2-4$ & $(400) 500-600$ \\
\hline
\end{tabular}

*Observado mediante estereoscopio. **Observaciones de Dorr y Wendt (2004).

ecuatorial, triangular o triangular convexo en vista polar y aberturas tricolporadas cortas (Rendón, 2005). Este conjunto de atributos estructurales apoyan su estrecha relación filogenética, ya sugerida, considerando caracteres moleculares (Nyffeler et al., 2005).

En los tricomas simples, las 3 categorías definidas resultaron de importancia taxonómica, dado que permiten reconocer 3 de las especies. Las categorías mediana y larga, presentan continuidad, no así la categoría corta presente exclusivamente en $M$. pentagonum y $M$. ruizii.
Los tricomas simples largos sólo se encontraron en $M$. hirsutum, única especie con hábito de arbusto o árbol pequeño, hojas de menor espesor, con nervios laterales mucho más cortos que en el resto de las especies, granos de polen con forma trilobulada y el lúmen más ancho, en vista polar (Rendón, 2005). En los tricomas fasciculados, las 2 categorías correspondientes con el largo de los brazos, permiten diferenciar a $M$. costaricense de $M$. vestitum; estas 2 especies presentaron una mayor similitud en cuanto al tipo de tricomas (principalmente simples 


\begin{tabular}{cccc}
\hline \multicolumn{4}{c}{ Número de tricomas $/ \mathrm{mm}^{2}$} \\
\hline $\begin{array}{c}\text { Longitud } \\
(\mu \mathrm{m})\end{array}$ & Base & Media & Ápice \\
\hline $\begin{array}{c}250-600 \\
--\end{array}$ & 13.14 & 3.73 & 1.15 \\
$100-150$ & 17.5 & 4.79 & 1.52 \\
$200-350$ & 6.59 & 2.48 & 0.77 \\
-- & & & \\
$150-300$ & $20 * *$ & $6 * *$ & $1 * *$ \\
$200-400$ & $\sim 0$ & $\sim 0$ & $\sim 0$ \\
$20-28$ & & & \\
-- & $\sim 0$ & $\sim 0$ & $\sim 0$ \\
$200-350$ &.. &.. &.. \\
$?$ & & & \\
-- & $>50 * *$ & $\sim 0$ & $\sim 0$ \\
$100-200$ & 15 & 12.5 & 12.5 \\
\hline & & & \\
\hline
\end{tabular}

y fasciculados). El estípite resultó menos pronunciado que lo anotado por Hardin (1990). Únicamente en $M$. uxpanapense se observaron tricomas estrellados con un número de brazos constante (8 y 10 brazos), en el resto de las especies el número de brazos varía arbitrariamente dentro de las amplitudes definidas.

Metcalfe (1989) señala que la humedad puede influir negativamente en la abundancia de tricomas. En $M$. guatemalense, la mayoría de los ejemplares presentó indumento glabrescente, pero el $13 \%$ del total resultó glabro, en estos casos los sitios de colecta se registraron en la ribera de ríos o arroyos, donde se espera un incremento notable en la humedad. Las especies con menos tricomas, M. pentagonum y $M$. uxpanapense, provienen de climas con alta humedad relativa. La primera especie fue colectada en remanentes de bosque mesófilo, en Alta Verapaz, Guatemala. En este sitio, Kunkel-Westphal y Kunkel (1979) registran una precipitación anual promedio de 3749 $\mathrm{mm}$. Mortoniodendron uxpanapense proviene de un clima similar, con precipitaciones promedio superiores a 4000 mm (Wendt, 1987). En los casos anteriores, se observan posibles relaciones entre la humedad y la densidad de tricomas; en $M$. guatemalense, la baja densidad, acentuada en algunos ejemplares, puede atribuirse a una alta humedad micro-climática. Para las especies $M$. pentagonum y $M$. uxpanapense, se considera un entorno más amplio, que también constituye un hábitat muy húmedo, lo cual podría relacionarse con la escasa densidad de tricomas presente en dichas especies. En M. guatemalense no se observan cambios en el indumento y tricomas por variaciones altitudinales (0-900 m), ni latitudinales (México, Guatemala, Costa Rica y Panamá), como se ha registrado para otras especies.

La presencia de indumento en el interior del fruto no es un rasgo exclusivo de $M$. guatemalense, como lo creía Miranda (1956), ya que se encontraron en otras 4 especies. En $M$. costaricense se observaron tricomas estrellados en el endocarpo, pero una mayor diversidad en la epidermis abaxial de la hoja donde predominaron los simples medianos y fasciculados cortos. No obstante, en M. anisophyllum, $M$. guatemalense, $M$. palaciosii y $M$. vestitum se observó el mismo tipo de tricomas en el endocarpo y en la epidermis abaxial de la hoja. Esta correspondencia resultó de acuerdo con la teoría apendicular del origen de los carpelos (Miranda, 1956), excepto en M. hirsutum, la cual presentó superficie abaxial glabrescente o axilar y endocarpo glabro. La presencia o ausencia de indumento puede relacionarse con la textura de la hoja, el tamaño del fruto y el número de estambres, puesto que las especies con endocarpo glabro (M. hirsutum, M. pentagonum, $M$. ruizii, $M$. uxpanapense) son de hoja coriácea o subcoriácea, con mayor número de estambres (54 a 85) y frutos 2 ó 4 veces más grandes y gruesos.

El indumento y el tipo de tricomas presentes en la superficie abaxial de las hojas y el endocarpo en el grupo revisado ayuda a identificar y clasificar morfológicamente las especies estudiadas; por ello, se presenta la siguiente clave para 9 especies de Mortoniodendron:

1. Envés de la hoja con más de un tipo de tricoma (simple, fasciculado, estrellado, capitado).

2. Envés de la hoja con 2 tipos de tricoma (simple, estrellado).

3. Envés de la hoja con indumento axilar

M. hirsutum

3. Envés de la hoja sin indumento axilar

M. pentagonum

2. Envés de la hoja con más de 2 tipos de tricoma (simple, fasciculado, estrellado, capitado).

4. Envés de la hoja sin tricomas capitados.

5. Venas de las hojas de orden mayor a $4 \sin$ indumento axilar.

6. Margen de la hoja sin tricomas.

7. Envés de la hoja con tricomas estrellados con más de 7 brazos M. guatemalense

7. Envés de la hoja con tricomas estrellados con menos de 7 brazos. M. costaricense

6. Margen de la hoja con tricomas.

M. vestitum 
5. Venas de las hojas de orden mayor a 4 con indumento axilar. M. anisophyllum

4. Envés de la hoja con tricomas capitados.

8. Endocarpo con tricomas M. palaciosii

8. Endocarpo sin tricomas M. ruizii

1. Envés de la hoja con un tipo de tricoma, estrellado corto M. uxpanapense

\section{Agradecimientos}

Esta investigación forma parte del tema de tesis del primer autor para obtener el grado de Maestría en Ciencias en Recursos Naturales y Desarrollo Rural. Se agradece a los curadores de los herbarios CHAPA, ENCB, MEXU y $\mathrm{XAL}$, por haber permitido revisar y remover material para el estudio, y al CONACYT por el financiamiento otorgado al primer autor para realizar estudios de maestría.

\section{Literatura citada}

Bamps, P. y A. Robyns. 1977. Westphalina macrocarpa gen. et sp. nov. (Tiliaceae) du Guatemala. Bulletin Jardin du Botanique National de Belgique 47: 183189.

Dickison, W. C. 2000. Integrative plant anatomy. Academic, San Diego, California. 533 p.

Dorr, L. y T. Wendt. 2004. A new species of Mortoniodendron (Malvaceae sens. lat.) from the rain forests of the Isthmus of Tehuantepec, México. Lundellia 7: 44-52.

Gual, M. 1998. La familia Tiliaceae Juss. en el estado de Guerrero. Tesis Maestría Facultad de Ciencias, UNAM, México, D.F. 70 p.

Hardin, J. 1990. Variation patterns and recognition of varieties of Tilia americana s.1. Systematic Botany 15: 33-48.

Hickey, L. 1988. A revised classification of the architecture of dicotyledonous leaves. In Anatomy of the dicotyledons, vol. 1, 2nd edition, C.R. Metcalfe y L. Chalk (eds.). Oxford University Press, Oxford, p. 25-39.

Kunkel-Westphal, I. y P. Kunkel. 1979. Litter fall in a Guatemalan primary forest, with details of leaf- shedding by some common tree species. Journal of Ecology 67: 665-686.

Lundell, C. L. 1965. Studies of tropical American plants. II. Mortoniodendron vestitum Wrightia 3: 117-125.

Metcalfe, C. R. 1989. Ecological anatomy and morphology. In Anatomy of the dicotyledons, vol.2, $2^{\text {nd }}$ edition, $\mathrm{C}$. Metcalfe y L. Chalk (eds.). Oxford University Press, Oxford, p. 126-151.

Miranda, F. 1956. El género Mortoniodendron y otros árboles notables de las selvas del sur de México. Anales del Instituto de Biología de la Universidad Nacional Autónoma de México 27: 321-336.

Nyffeler, R., C. Bayer, W. S. Alverson, A. Yen, B. A. Whitlock, M. W. Chase y D. A. Baum. 2005. Phylogenetic analysis of the Malvadendrina clade (Malvaceae s.l.) based on plastid DNA sequences. Organisms, Diversity and Evolution 5: 109-123.

Standley, P. C. 1942. Tiliaceae. In Contributions toward a flora of Panamá. VI. Collections chiefly by H. von Wedel in Bocas del Toro, R. E. Woodson Jr. y R. W. Schery (eds.). Annals of the Missouri Botanical Garden 29:317-379.

Standley, P. y J. Steyermark, 1940. Studies of tropical American plants. XI. Field Museum of Natural History, Botanical Series 22: 133-218.

Standley, P. C. y J. Steyermark, 1949. Flora of Guatemala. Fieldiana Botany 24(6): 302-324.

Rendón C., N. 2005. Revisión del género Mortoniodendron (Tiliaceae) en México. Tesis Maestría El Colegio de la Frontera Sur, San Cristóbal de las Casas, Chiapas. $94 \mathrm{p}$.

Theobald W. L., J. L. Krahulik y R. C. Rollins. 1988. Trichome description and classification In Anatomy of the dicotyledons, vol. $1,2^{\text {nd }}$. edition, R. Metcalfe $y$ L. Chalk (eds.). Oxford University Press, Oxford, p. 40-53.

Valencia, S. y A. Delgado. 2003. Los tricomas foliares en la caracterización de un grupo de especies del género Quercus, sección Lobatae (Fagaceae). Anales del Instituto de Biología, Universidad Nacional Autónoma de México, Serie Botánica 74: 5-15.

Wendt, T. 1987. Las selvas de Uxpanapa, Veracruz-Oaxaca, México: evidencia de refugios florísticos cenozoicos. Anales del Instituto de Biología, Universidad Nacional Autónoma de México, Serie Botánica 58: 29-54. 